\title{
Changes in cytoplasmic and extracellular neuromelanin in human substantia nigra with normal aging
}

\author{
Dmitrii E. Korzhevskii, Olga V. Kirik, Valeriia V. Guselnikova, Darya L. Tsyba, Elena A. Fedorova, \\ Igor P. Grigorev
}

Department of General and Special Morphology, Institute of Experimental Medicine, Saint Petersburg, Russian Federation

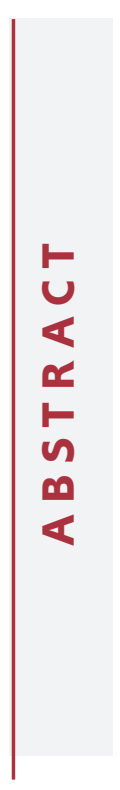

\begin{abstract}
Neuromelanin (NM) is a dark polymer pigment produced in certain populations of catecholaminergic neurons in the brain. It is present in various areas of the human brain, most often in the substantia nigra (SN) pars compacta and the locus coeruleus, the main centers of dopaminergic and noradrenergic innervation, respectively. Interest in NM has revived in recent years due to the alleged link between NM and the particular vulnerability of NM-containing neurons to neurodegeneration. The aim of this work was to study the structural, cytochemical, and localization features of cytoplasmic and extracellular NM (eNM) in the human SN pars compacta during normal aging. Sections of human SN from young/middle-aged adults (25 to 51 years old, $n=7$ ) and older adults (60 to 78 years old, $n=5$ ), all of which had no neurological disorders, were stained histochemically for metals (Perls' reaction, Mayer's hematoxylin) and immunohistochemically for tyrosine hydroxylase (TH), Iba1, and CD68. It was shown that dopaminergic neurons in SN pars compacta differ in the amount of NM and the intensity of TH-immunoreactivity. The number of NM-containing neurons with decreased TH-immunoreactivity positively correlates with age. eNM is present in SN pars compacta in both young/middle-aged and older adults. The number of eNM accumulations increases with aging. Cytoplasmic and eNM are predominantly not stained using histochemical methods for detecting metals in people of all ages. We did not detect the appearance of amoeboid microglia in human SN pars compacta with aging, but we found an age-related increase in microglial phagocytic activity. The absence of pronounced microgliosis, as well as a pronounced loss of NM-containing neurons, indicate the absence of neuroinflammation in human SN pars compacta during normal aging.
\end{abstract}

Key words: neuromelanin; substantia nigra; human; aging; microglia; Iba-1; Perls' reaction.

Correspondence: Valeriia Guselnikova, Department of General and Special Morphology, Institute of Experimental Medicine, Acad. Pavlov Street, 197376 Saint Petersburg, Russian Federation. Tel. +7.9817568105. E-mail: Guselnicova.Valeriia@yandex.ru

Contributions: DEK, conceptualization and design of the study, confocal microscopy, review and editing of the manuscript; OVK, immunohistochemistry, microscopic investigation; VVG, immunohistochemistry, confocal microscopy, microscopic investigation original draft preparation; DLT, immunohistochemistry, microscopic investigation, statistical analysis; EAF, immunohistochemistry, microscopic investigation; IPG, conceptualization of the study, review and editing of the manuscript. All authors have read and agreed to the published version of the manuscript.

Funding: This work was supported by the funds of the Institute of Experimental Medicine, St. Petersburg, Russian Federation.

Conflict of interest: The authors declare no conflict of interest.

Ethical approval: The study was conducted in accordance with the Declaration of Helsinki of 1975, and the protocol was approved by the local Ethics Committee of the Institute of Experimental Medicine, St. Petersburg, Russian Federation (protocol \#58-9/1-684, December 11, 2009).

Availability of data and materials: The data generated and analyzed in this study are available from the corresponding authors on reasonable request. 


\section{Introduction}

Melanins are the natural dark pigments that include different types of melanin found in bacteria, fungi, invertebrate and vertebrate animals. In mammals, three types of melanin are known; these are eumelanin, pheomelanin and neuromelanin (NM). Pheomelanin and eumelanin are peripheral pigments implicated in coloration of hair, skin and iris. NM is found in the central nervous system, primarily in primates including humans. It is located in various brain areas, most abundantly in the substantia nigra (SN) pars compacta and locus coeruleus, the major brain centers of dopaminergic and noradrenergic innervation, respectively. ${ }^{1}$ In normal adult human SN pars compacta, NM is densely packed in the cytoplasm of most neurons.

The structure and synthesis of NM remains partly understood. The problem is, at a great extent, because the pigment is an amorphous insoluble complex substance with inconsistent composition. Since NM is most often seen in catecholaminergic (dopamine- or noradrenaline-containing) cells, it is logical to assume that catecholamines are involved in the synthesis of the pigment. This view is supported by the finding that tyrosine (amino acid, the precursor of both dopamine and norepinephrine) and enzyme tyrosinase are involved in the synthesis of peripheral melanins, the eumelanin and pheomelanin. ${ }^{2}$ However, tyrosinase has not been found in the human brain (particularly, the substantia nigra) or found in negligible amounts. ${ }^{3,4}$ Therefore, NM synthesis via spontaneous nonenzymatic dopamine auto-oxidation has been supposed, ${ }^{5}$ although involvement of tyrosinase or any other enzyme in production of NM cannot be excluded. ${ }^{6}$ Derivatives of catecholamines copolymerize with cysteine, some proteins, lipids and aliphatic compounds producing a complex macromolecule with varying set of components. ${ }^{7-10}$ Moreover, NM can absorb metals, such as iron, copper, manganese, lead and mercury, some drugs and chemicals including toxic compounds. ${ }^{11-18}$ These data suggest that NM may serve a neuroprotective function as a chelator for toxic metals and chemicals.

The other side of the coin is that accumulation of potentially toxic compounds in NM granules is a risk factor. Indeed, if NMcontaining neurons degenerate (whatever the cause), the NM granules leaks into the intercellular space, and dangerous metals and substances can injure other nerve cells. Particularly, iron released from NM can contribute to formation of cytotoxic free radicals originating from elevated levels of $\mathrm{H}_{2} \mathrm{O}_{2}$ via Fenton reaction. ${ }^{19}$ Furthermore, eNM can activate the CNS resident immune cells, microglia and, in this way, induces neuroinflammation that can result in neurodegeneration. ${ }^{20-25}$ Considering this properties of NM, its direct or indirect implication in degeneration of dopaminergic neurons in the SN and, consequently, it is possible role in the etiology and pathogenesis of Parkinson's disease has been suggested. ${ }^{23,25-30}$ The present study aims to analyze the structural, cytochemical and localization features of intra- and eNM in human SN pars compacta during normal aging.

\section{Materials and Methods}

\section{Human brain specimens}

Midbrain fragments of neurologically normal individuals ( $n=12$, males and females, ages 25 to 78 years) were used in this work. Individuals were categorized by age into young/middle-aged adults (25-51 years, $n=7)$ and older adults (60-78 years, $n=5)$.
Paraffin blocks of 1-1,5 $\mathrm{cm}^{3}$ (10x10x10-mm) were obtained from the archive of Department of General and Special Morphology, Institute of Experimental Medicine (St. Petersburg, Russia). Initially the autopsy tissue was fixed in the zinc-ethanol-formalin ${ }^{31}$ in a ratio of 1:50 (material:fixative). For morphological analysis, 5 $\mu \mathrm{m}$ thick sections were cut using rotary (RM 2125RT, Leica, Germany) or sliding (Leica SM 2000R, Leica, Germany) microtome and mounted on poly-L-lysine-coated (Polysine ${ }^{\mathrm{TM}}$, MenzelGläser, Germany) glass slides.

\section{Histochemistry}

Mallory's staining with hematoxylin ${ }^{32}$ and Perls' reaction ${ }^{33}$ were used to reveal metal compounds.

\section{Immunohistochemistry}

Before immunohistochemistry study the sections were deparaffinized and rehydrated routinely. For better antigen detection, the heat-induced antigen retrieval in modified citrate buffer, pH 6.1 (S1700, Agilent-Dako, Santa Clara, CA, USA) was performed in a conventional steamer for $25 \mathrm{~min}$. Endogenous peroxidase was blocked in a 3\% aqueous solution of hydrogen peroxide for $10 \mathrm{~min}$. The preparations were pretreated with the blocking solution (Protein Block, Spring Bioscience, Pleasanton, CA, USA) for $10 \mathrm{~min}$ at room temperature. After that anti-tyrosine hydroxylase rabbit polyclonal (Abcam, Cambridge, UK; ab112; 1:1000), anti-Iba-1 protein (AIF1) goat polyclonal (1:200, ab5076, Abcam, UK), and anti-CD68 mouse monoclonal (clone PG-M1, ready-touse, N1576, Dako, Glostrup, Denmark) primary antibodies were applied. To visualize the applied primary antibodies the following reagents were used: for rabbit antibodies, Reveal Polyvalent HRP DAB (Spring Bioscience); for goat antibodies, biotinylated antigoat antibody (Agilent-Dako) and streptavidin conjugated with HRP (streptavidin/horse radish peroxidase; Spring Bioscience); for mouse antibodies, MACH 2 Mouse HRP-Polymer (BioCare Medical, Pacheco, CA, USA). The peroxidase label was detected using diaminobenzidine chromogen (DAB+; Agilent-Dako). After immunocytochemical reactions, some sections were post-stained with hematoxylin. For all immunohistochemical reactions, the control reactions recommended by the antibody manufacturer were performed.

\section{Microscopy}

The sections were examined in transmitted light under a Leica DM750 (Leica, Wetzlar, Germany) microscope and photographed using ICC50 camera (Leica). Images were processed using ImageJ (NIH, USA) software. Neurons and extracellular melanin were counted in 12 fields of view with an area of $0.08 \mathrm{~mm}^{2}$.

To detect autofluorescence of NM and lipofuscin in brain tissue, unstained sections enclosed in a permanent medium were used. The sections were examined under LSM710 confocal laser microscope (Carl Zeiss, Jena, Germany) using lasers with wavelengths of $405 \mathrm{~nm}$ and $561 \mathrm{~nm}$ and Zen-2012 (Carl Zeiss) software.

\section{Statistical analysis}

Statistical analysis was conducted using RStudio v1.2 software (http://www.rstudio.com/). Age groups and morphometric data were analyzed. Continuous variables are presented as means and standard deviations (SD). Differences between age groups were assessed using the unpaired Mann-Whitney test. Correlation between morphometric data and age was analyzed with the Spearman rank correlation test. The $\mathrm{p}<0.05$ was considered statistically significant. 


\section{Results}

Tyrosine hydroxylase and NM distribution in neurons of the human SN pars compacta

The light microscopic analysis revealed that the SN pars compacta contains densely-packed tyrosine hydroxylase (TH)- immunoreactive (IR) dopaminergic neurons (Figures 1A and 2 $\mathrm{D}, \mathrm{E})$. TH-immunostaining was localized to perikaryon and processes of the neurons. Cytoplasm of dopaminergic neurons was filled with NM granules. The neurons of SN pars compacta differed according to their amount of cytoplasmic NM and THimmunoreactivity. Most neurons have numerous densely packed $\mathrm{NM}$ granules in perikaryon. TH-immunoreactivity of these neurons was either intensive (Figure 1A, arrow 1), or medium/weak

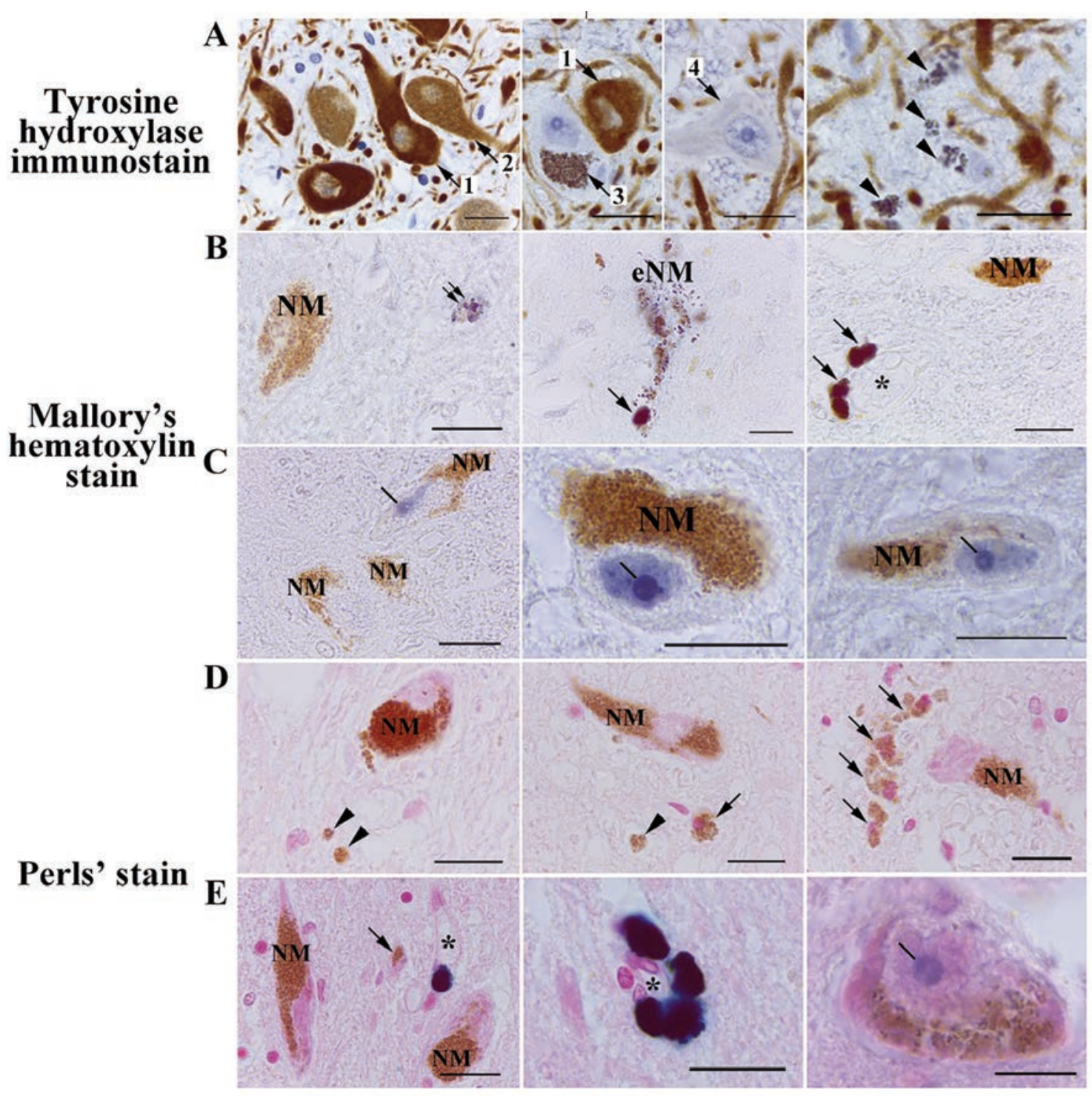

Figure 1. NM-containing neurons and eNM in human SN pars compacta. A) Tyrosine hydroxylase immunostaining counterstained with hematoxylin. B,C) Mallory's hematoxylin staining. D,E) Perls' staining. A) Most neurons in human SN pars compacta have NM in the cytoplasm and are either intensely TH-immunoreactive (arrow 1) or moderate/weak TH-immunoreactive (arrow 2); some of neurons in SN pars compacta are NM-containing and TH-immunonegative (arrow 3); single SN pars compacta neurons are TH-immunonegative and do not contain NM (arrow 4). The majority of human SN pars compacta samples analyzed contain accumulations of eNM (arrowhead). B,C) Intracellular NM does not react with Mallory's hematoxylin, remaining golden brown; dark blue are small, fine-grained clusters in the neuropil (double arrow), destroyed neurons (extracellular neuromelanin,- eNM), and macrophages (arrow); in 2 cases excluded from the initial samples due to prolonged illness of subjects, we found intense blue staining of the nucleus and intranuclear structures in some of the dopaminergic neurons in $\mathrm{SN}$ pars compacta $(\mathrm{C}$, line). D,E) After Pearls' reaction for iron, no staining of intracellular NM and eNM (arrowhead) was observed; some parenchymal macrophages contained golden brown granules in the cytoplasm (arrow); some perivascular macrophages expressed dark blue staining of the cytoplasm due to the presence of the pigment hemosiderin, which contains iron oxide; the asterisk is the lumen of the blood vessel; as with the use of Mallory's hematoxylin, in 2 cases, the nucleolus of some SN pars compacta neurons was stained blue (line), which indicates the accumulation of non-heme iron in the nucleoli of these cells. Scale bar: $20 \mu \mathrm{m}$. 
(Figure 1A, arrow 2). Quantitative analysis showed that the proportion of neurons with reduced $\mathrm{TH}$-immunoreactivity increases with age (Figure 2A). In young/middle-aged adults, the percentage of such neurons averages $15.6 \%$ (SD 10.6), while in older adults it is $40.2 \%$ ( $\mathrm{SD} 19.5$ ), which do not reach statistical significance $(\mathrm{W}=29, \mathrm{p}=0.07)$. Correlation analysis revealed a positive relationship between age and the number of NM-containing neurons with decreased TH-immunoreactivity $(\mathrm{r}=0.6, \mathrm{p}=0.04)$.

In addition to NM-containing TH-IR neurons, SN pars compacta contains NM-filled TH-immunonegative neurons (Figure $1 \mathrm{~A}$, arrow 3 ). Their number in young/middle-aged people ranges from $3 \%$ to $29 \%$ (mean 5.6 , SD 10.5 ), in old people - from $3 \%$ to $16 \%$ (mean 7, SD 6.4), which is statistically insignificant difference $(\mathrm{W}=11.5, \mathrm{p}=0.36)$ (Figure 2B). Finally, few TH-immunonegative neurons that do not contain NM were found in the SN pars compacta (Figure 1A, arrow 4). They were rare in all studied samples and had no signs of degeneration.

\section{eNM in the human SN pars compacta}

In 10 out of 12 analyzed cases, accumulations of eNM were detected in human SN pars compacta (Figures $1 \mathrm{~A}$ and $2 \mathrm{~F}$, arrowhead). Quantification has shown that eNM accumulations are rare in young/middle-aged people. Their average quantity is 6 per $\mathrm{mm}^{2}$ tissue (SD 6). On the contrary, in old people, accumulations of eNM are often detected. Their average number is 29 per $\mathrm{mm}^{2}$ tissue (SD 35), which was statistically insignificant in comparison to people of young/middle age $(\mathrm{W}=28, \mathrm{p}=0.1)$ (Figure $2 \mathrm{C})$. However, correlation analysis showed a positive relationship between the age and the number of eNM accumulations $(r=0.63, p=0.03)$.

\section{Staining with Mallory's hematoxylin}

In sections stained with Mallory's hematoxylin, eNM was never bound the stain, retaining its golden-brown color (Figure 1 B,C, NM). We also did not find the appearance of intracellular NM staining with age. We observed medium-sized fine-grained clusters stained in a dark color in the SN pars compacta parenchyma (Figure 1B, double arrow), but it is not possible to determine whether they are eNM aggregations or cytoplasmic inclusions of some cells. In one of the investigated cases (woman, 74-year-old), degenerating neurons with destructive NM stained darkly were identified (Figure 1B, eNM). In the area of the destructive NM, a dark-colored cell was present, which is probably a macrophage phagocytizing eNM. We also noted intense dark staining of the cytoplasm of perivascular macrophages in the SN pars compacta of most of the samples studied (Figure 1B, arrow).

Interestingly, in 2 cases excluded from the initial samples due to prolonged illness of subjects (man, 27 years old, pneumonia; woman, 87 years old, oncology), we found intense blue staining of the nucleus and intranuclear structures in some of the dopaminergic neurons in SN pars compacta (Figure 1C). The nucleolus was stained especially intensely in these neurons, with the formation of an unstained region of the giant fibrillar center (Figure 1C, line). In some neurons, numerous stained microstructures were also present in the nucleoplasm.

\section{Pearls' reaction for iron}

NM accumulates iron and, thus, inactivates this redox-active metal. ${ }^{11}$ Therefore, we carried out the Pearls' reaction for ferric iron. Pearls' reaction for ferric iron failed to reveal staining of both intracellular NM (Figure 1 D,E, NM) and eNM (Figure 1D, arrowhead). All NM remained golden brown in both young/middle aged and older subjects. Cytoplasm of some perivascular macrophages was stained dark blue (Figure 1E, dark blue stain). This is probably due to the accumulation of the hemosiderin pigment, which contains iron oxide, in the cytoplasm of macrophages. As with the use of Mallory's hematoxylin, we found blue staining of the nucleolus of some SN pars compacta neurons in 2 cases (Figure 1D, line) excluded from the initial samples due to prolonged illness of subjects.

\section{Separate imaging of NM and lipofuscin pigments}

Interestingly, in some cases, small cells were found in the parenchyma, the cytoplasm of which was filled with golden brown granules similar to NM (Figure 1D, arrow). These are probably macrophages that phagocytosed eNM or accumulated lipofuscin pigment in the cytoplasm, which is morphologically similar to NM. Lipofuscin can be distinguished from NM using fluorescence or confocal laser microscopy. A characteristic feature of lipofuscin is autofluorescence. We suggest using $561 \mathrm{~nm}$ laser to excite lipofuscin autofluorescence (Figure 3, L). The maximum emission is between 580 and $680 \mathrm{~nm}$. Using a $405 \mathrm{~nm}$ laser to excite autofluorescence in neural tissue can enhance contrast. Unlike lipofuscin, NM is not autofluorescent (Figure 3, NM).

\section{The state of microglia in the human SN during normal aging}

Next, we studied the response of microglia to the presence of eNM in SN pars compacta (Figure 4). Using Iba-1 immunostaining, we did not detect the activation of microglia in SN pars compacta with age. In both young/middle-aged people and older adults, Iba-1-immunopositive microglia were evenly distributed over the entire SN pars compacta area and did not form clusters in any of the studied samples. Microglia had a ramified phenotype in both young/middle-aged and older adults (Figure 4A). The processes of microglia often approached the bodies of NM-containing neurons, contacting and surrounding them (Figure 4B). There were rare microgliocytes with an enlarged body and/or less branched processes in all cases. We did not find amoeboid microglia in SN pars compacta. In both age groups, the presence of Iba-1-immunoreactive perivascular macrophages localized near blood vessels was noted (Figure 4C). We did not observe the presence of activated microglia around clumps of eNM. Iba-1immunoreactive microglia near such clusters always had a normal ramified morphology (Figure 4D).

Interestingly, age-dependent differences in CD68 staining in human SN pars compacta were found (Fig. 4E-F). There were only CD68-immunopositive perivascular macrophages in the SN pars compacta of young/middle-aged people (Figure 4E, black arrowhead) and no CD68-immunostaining in the brain parenchyma (Figure 4E). In contrast, SN pars compacta of older adults contained a large amount of discrete CD68-immunopositive granules (Figure 4F, red arrowhead). Sometimes CD68-immunopositive aggregates formed profiles of ramified cells (Figure 4F, red arrow). Similar to Iba-1 staining, we did not detect microglia with amoeboid morphology when immunostained for CD68.

\section{Discussion}

$\mathrm{NM}$ is a byproduct of the metabolism of dopamine or norepinephrine and is formed during the synthesis of these neurotransmitters in catecholaminergic neurons. In this context, our detection of NM-free neurons in human SN pars compacta may result from the presence of nondopaminergic neurons in this region. For example, these can be cholinergic neurons. ${ }^{34}$

In humans, NM is first detected in early childhood and gradually accumulates with age until it occupies most of the cytoplasm of neurons, since neurons do not appear to have mechanisms to destroy or remove this pigment. ${ }^{6,35} \mathrm{NM}$ synthesis is believed to be neuroprotective, as it removes excess cytosolic catechins and their 
derivatives, and chelates toxic metals. ${ }^{36,37}$ The recent development of a murine model producing human NM allowed for the first time the experimental evaluation of the effects of age-related accumulation of NM to levels achieved in the elderly in vivo. The use of this model has shown that the progressive intracellular accumulation of NM that occurs with age ultimately induces PD-like neuronal dysfunction and neurodegeneration once a certain threshold of NM accumulation is reached. Therefore, while NM synthesis itself may be neuroprotective, its long-term accumulation has detrimental effects. ${ }^{6}$ This is in good agreement with our data on a decrease in the TH-immunoreactivity of dopaminergic neurons in $\mathrm{SN}$ pars compacta with age. Probably, the found presence of neurons with different degrees of TH-immunoreactivity reflects the difference in the functional state of dopaminergic neurons in human SN pars compacta. The results obtained indicate a gradual decrease in the functional activity of dopaminergic neurons with aging.

After the death of NM-containing neurons, NM is released into the extracellular space. Large amounts of eNM are observed in the brains of older adults when compared to the brains of younger adults. ${ }^{20}$ In agreement with these data, we found a positive correlation between age and the amount of eNM in the human SN pars compacta. Increased amount of eNM is thought to stimulate dysfunction and degeneration of the remaining NM-containing neurons. ${ }^{38}$ In our study, we noted an age-dependent decrease in THimmunoreactivity of dopaminergic neurons, which may indicate the development of dysfunction. But we did not find a pronounced loss of NM-containing neurons in SN pars compacta with aging.

One of the difficulties in studying eNM by light microscopy is its morphological similarity with intracellular accumulations of lipofuscin. Lipofuscin is a protein- and lipid-based pigment whose
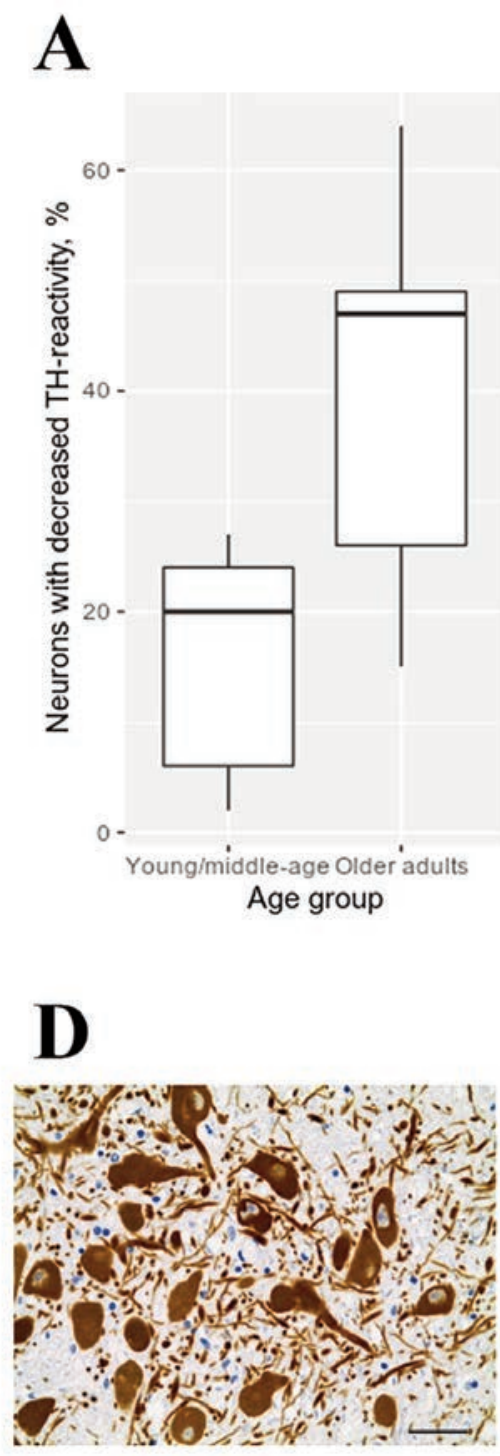
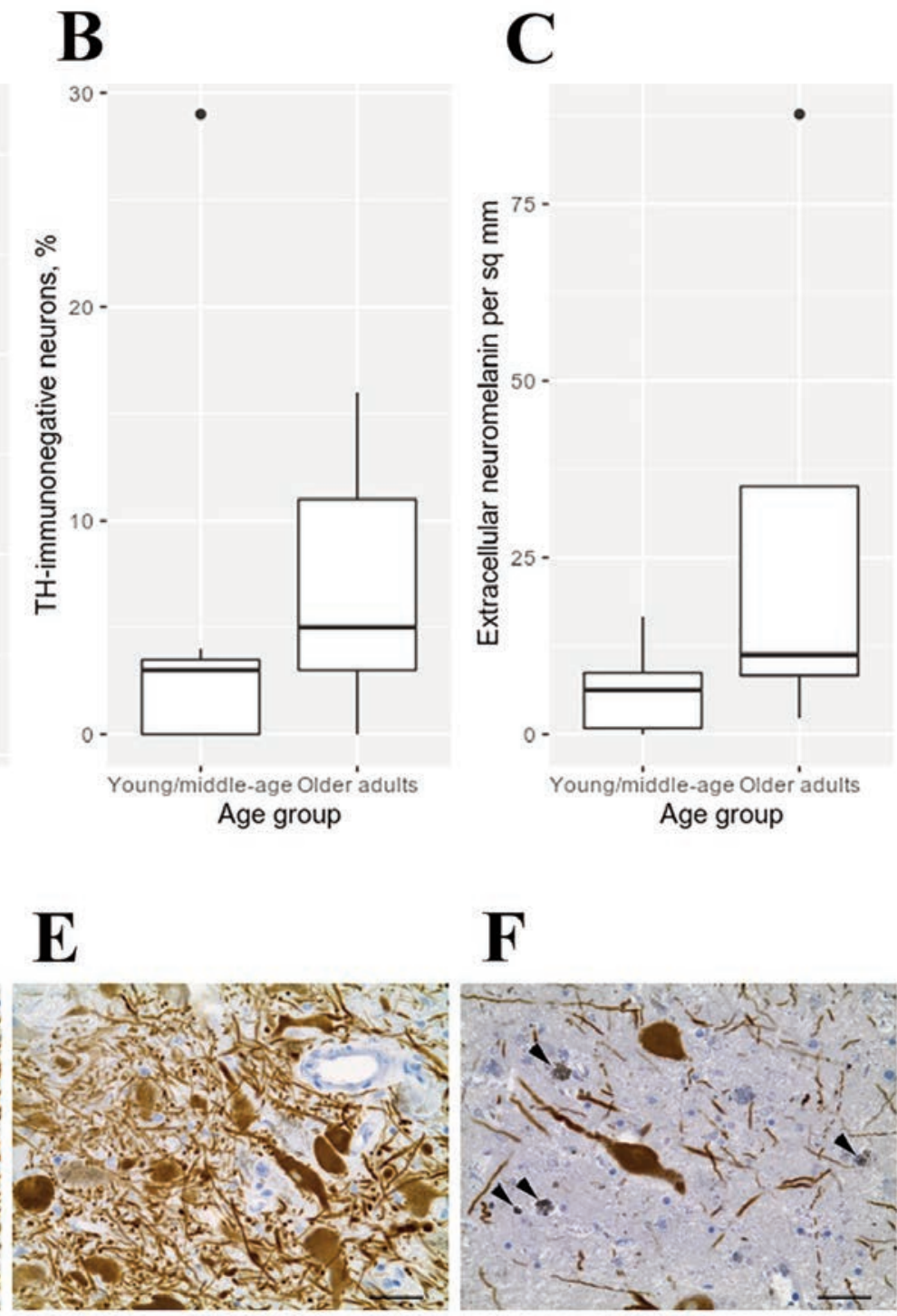
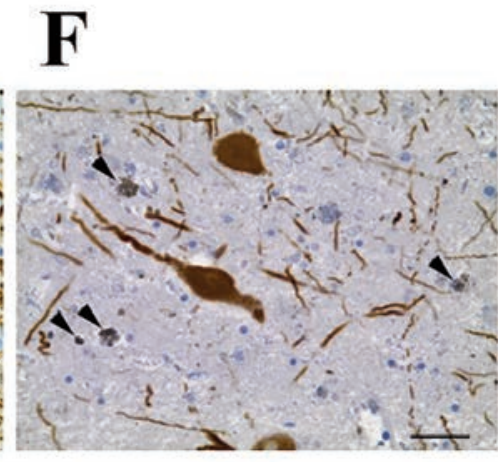

Figure 2. Distribution of morphometric characters in SN pars compacta depending on age. A) Proportion of neurons with decreased TH-immunoreactivity. B) Proportion of TH-immunonegative neurons. C) Amount of eNM per sq mm; continuous variables are presented as means and standard deviations (SD); differences between age groups were assessed using the unpaired Mann-Whitney test; black dots are outliers. D-F) Tyrosine hydroxylase immunostaining counterstained with hematoxylin. D) 25-year-old men; most neurons in human SN pars compacta are intensely TH-immunoreactive, intracellular NM accumulations are absent. E) 62-year-old women; most neurons in human $\mathrm{SN}$ pars compacta are moderate/weak TH-immunoreactive; some of neurons are TH-immunonegative F) 74year-old women; eNM (arrowhead) is found in large amount. Scale bar: $20 \mu \mathrm{m}$. 
accumulation in neurons is associated with post-mitotic cellular aging. Its amount increases with age. Lipofuscin granules accumulate mainly in the perinuclear region, but can be present throughout the perikaryon, in dendrites, axons, and even in presynapses. ${ }^{39}$ Lipofuscin is also produced by glial cells, but since these cells, unlike neurons, continue to divide, the amount of lipofuscin is diluted via division. The high concentration of lipofuscin in glial cells is the result of their uptake of neuronal lipofuscin..$^{39,40}$ In present study, we propose the use of confocal microscopy to distinguish between NM and lipofuscin in human SN pars compacta. Unlike lipofuscin, NM is not autofluorescent when excited with a $561 \mathrm{~nm}$ laser. This approach can be applied to more accurately identify the two pigments.

According to modern concepts, NM is an insoluble and degradation resistant complex polymeric compound. It consists of a mixture of derivatives of oxidized catecholamines, lipids, peptides and metals that accumulate in autophagosomes and/or lysosomes of catecholaminergic neurons. Based on data on the accumulation of metals in $\mathrm{NM},{ }^{37}$ we estimated the presence of metals in human SN pars compacta using staining with Mallory's hematoxylin. The essence of the technique is that a hematoxylin solution is prepared without the addition of metal salts. When sections are treated with such hematoxylin, it reacts with structures containing copper and/or iron, staining them blue or black. ${ }^{32}$ Unfortunately, we did not detect dark staining of intracellular and/or eNM when using Mallory's hematoxylin, which may be due to insufficient sensitivity of the method.

Iron is a most typical metal for NM, which accumulates in large quantities. Iron and other redox-active metals are inactivated by interaction with NM, and it has been suggested that the chelating potential of NM allows the cell to inactivate potentially toxic metal ions. ${ }^{11}$ We did not detect NM staining using the Perls' reaction. Similar results were obtained earlier in the study of SN pars compacta of patients with Parkinson's disease. ${ }^{41}$ Classical Perls' staining in this case showed that in Parkinson's disease patients, $\mathrm{Fe}^{3+}$ accumulates mainly in macrophages. Its presence has also been noted in astrocytes, reactive microglia, unpigmented neurons, and damaged areas devoid of pigmented neurons. NM in neuronal cytoplasm and eNM were not stained by Perls' reaction. ${ }^{41}$ However, other methods have repeatedly shown the presence of large amounts of iron in NM-containing SN neurons in norm and

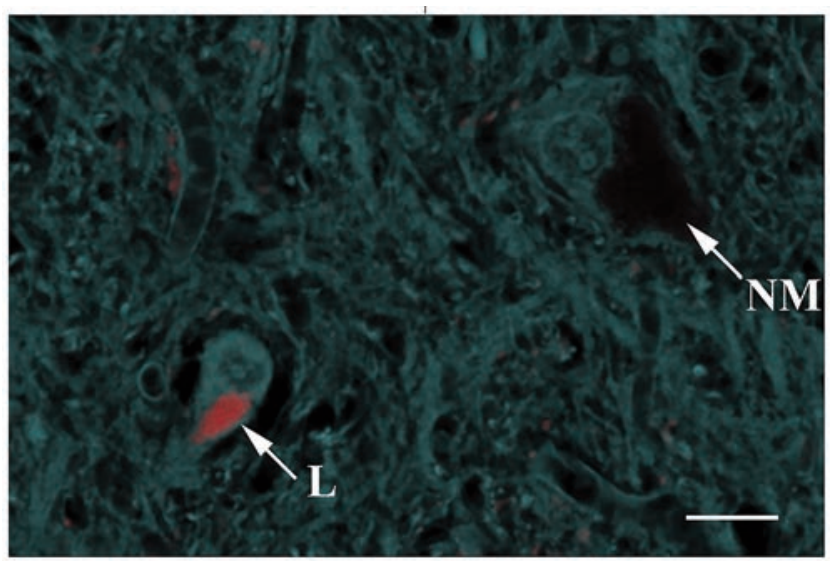

Figure 3. NM-containing neuron and lipofuscin-containing neuron in SN pars compacta of 73-year-old men. The lipofuscin pigment (L) appears fluorescent. Excitation with the yellow (561 $\mathrm{nm})$ laser. Neuromelanin (NM) within a neuron is non-fluorescent. Autofluorescence of nerve tissue upon excitation with $\mathbf{4 0 5}$ $\mathrm{nm}$ laser. Laser scanning microscopy. Scale bar: $20 \mu \mathrm{m}$.
Parkinson's disease. Thus, Zecca et al. showed that NM is the main storage of iron in SN neurons in normal humans, and its content increases 100 -fold during aging. ${ }^{37,42}$ Several precise methods with a subcellular resolution such as laser microprobe mass analysis and energy dispersive X-ray microanalysis have demonstrated an accumulation of iron in NM granules in the SN pars compacta of $\mathrm{PD}$ patients. ${ }^{43,44}$ Application of Mössbauer spectroscopy showed that $\mathrm{SN}$ contains high-spin ferric iron, which is in a ferritin-like ironoxyhydroxide cluster form. ${ }^{42}$ Negative results of NM staining with Perls' reaction may be associated with insufficient sensitivity of the method. Probably, the iron content in NM is insufficient for detection by histochemical method. Iron can be detected using the classical Perls' method only at a significant concentration, as in the case of hemosiderin in macrophages.

When using either of both, Mallory's hematoxylin and Perls' reaction, we found blue staining of the nucleolus of some SN pars compacta neurons in 2 cases excluded from the initial samples due to prolonged illness of subjects. This indicates the pathologic accumulation of non-heme iron in the nucleoli of dopaminergic neurons of human SN pars compacta. The presence of iron in the nucleoli of NM-containing neurons of human SN pars compacta was described in this Laboratory earlier. ${ }^{45,46}$ Similar data were recently obtained for nerve cells in the rat brain. Using a nuclear microprobe and scanning proton induced X-ray emission spectrometry Reinert et al. conducted a quantitative analysis of the concentration of iron in neurons and glial cells of different brain areas in rats, and showed that the nucleolus has a higher concentration of iron than the nucleus and cytoplasm of neurons.$^{47}$ It is known that the nucleolus is the site of synthesis of ribosomal RNA (rRNA) and the site of assembly of ribosomal subunits. Iron can also be a cofactor in some nucleolar proteins. Roschzttardtz et al. suggested that iron may be involved in rRNA metabolism. ${ }^{48}$ Moreover, ferritin binds to $\mathrm{DNA}^{49}$ and can concentrate iron in the nucleolus. Another source may be iron, which is directly bound to RNA. The ironbinding capacity of rRNA is higher than that of mRNA or tRNA,,$^{50}$ which leads to higher iron concentrations in the nucleolus. For Alzheimer's disease, it has been shown that rRNA is oxidized under the influence of bound iron, ${ }^{50}$ that is, the nucleolus may be especially vulnerable to oxidative activity.

According to the literature, the extraneuronal NM is associated with microglial activation. ${ }^{14,22}$ The contribution of eNM to the development of neuroinflammation and death of dopaminergic neurons in SN is widely discussed in the context of PD. eNM is insoluble, contains a large amount of reactive iron, and in PD it can exist in the extracellular spaces in large quantities and for a long time. ${ }^{37,51}$ Therefore, NM released from dying neurons is a strong candidate for an agent that induces chronic inflammation in SN. By itself, eNM is not toxic to neurons. However, in vitro and in vivo studies have shown the ability of eNM to activate microglia. ${ }^{22,23,25}$ Thus, the addition of human NM to microglia cultures was noted to activate positive chemotaxis, the production of superoxide, nitric oxide, hydrogen peroxide, as well as the release of tumor necrosis factor- $\alpha$, interleukin- 6 and NO. The release of inflammatory molecules and cytotoxic agents by NM-activated microglia promotes damage and degeneration of dopaminergic neurons. In vivo $\mathrm{NM}$ injected into rat $\mathrm{SN}$ induces microgliosis and death of dopaminergic neurons. ${ }^{22,23}$ Given that there is a continuous release of NM by dying neurons in $\mathrm{SN}$ in PD, it has been suggested that $\mathrm{eNM}$ can cause sustained activation of microglia and, as a consequence, neurodegeneration in $\mathrm{PD} .{ }^{24}$

The question remains open whether similar processes develop in human SN during normal aging. Since the amount of eNM in the $\mathrm{SN}$ increases with aging, it seems logical to expect a significant activation of microglia in the SN in the elderly. Previously, Beach et al. reported a pronounced microglial response in $\mathrm{SN}$ of elderly 
people without neurological disease. ${ }^{20}$ They described the presence of hypertrophied MHC Class II - immunoreactive cells in the SN of the elderly. These cells were especially numerous around the extracellular deposits of NM. Sometimes the processes of microglia, as well as rounded amoeboid cells, contained phagocytosed NM. ${ }^{20}$

In our study, we found no morphological signs of microglia activation against the background of an increase in the amount of eNM in SN in older adults. Similar to our findings, another study also stated no significant correlation between normal aging and microglial activation in human SN pars compacta. ${ }^{52}$ Jyothi et al. found that the number of microglia does not significantly increase with age in any of the human SN pars compacta regions.
Morphologically activated microglia are rare ${ }^{52} \mathrm{~A}$ study in primates also did not show a significant correlation between normal aging and the amount of microglia in the SN pars compacta. ${ }^{53}$ Probably, eNM is not the main factor in the activation of microglia, and in the case of $\mathrm{PD}$, the pronounced microglial response is not directly associated with an increase in the amount of eNM.

Importantly, we observed an increase in CD68 immunoreactivity with age in human SN pars compacta. CD68 is a lysosomal marker of phagocytic activity, mainly localized in microglia and perivascular macrophages. Although there is some CD68 expression on resting microglia, it is commonly considered a marker of activated phagocytic microglia. ${ }^{54,55}$ However, we did not detect CD68-immunopositive microglia with amoeboid morphology in
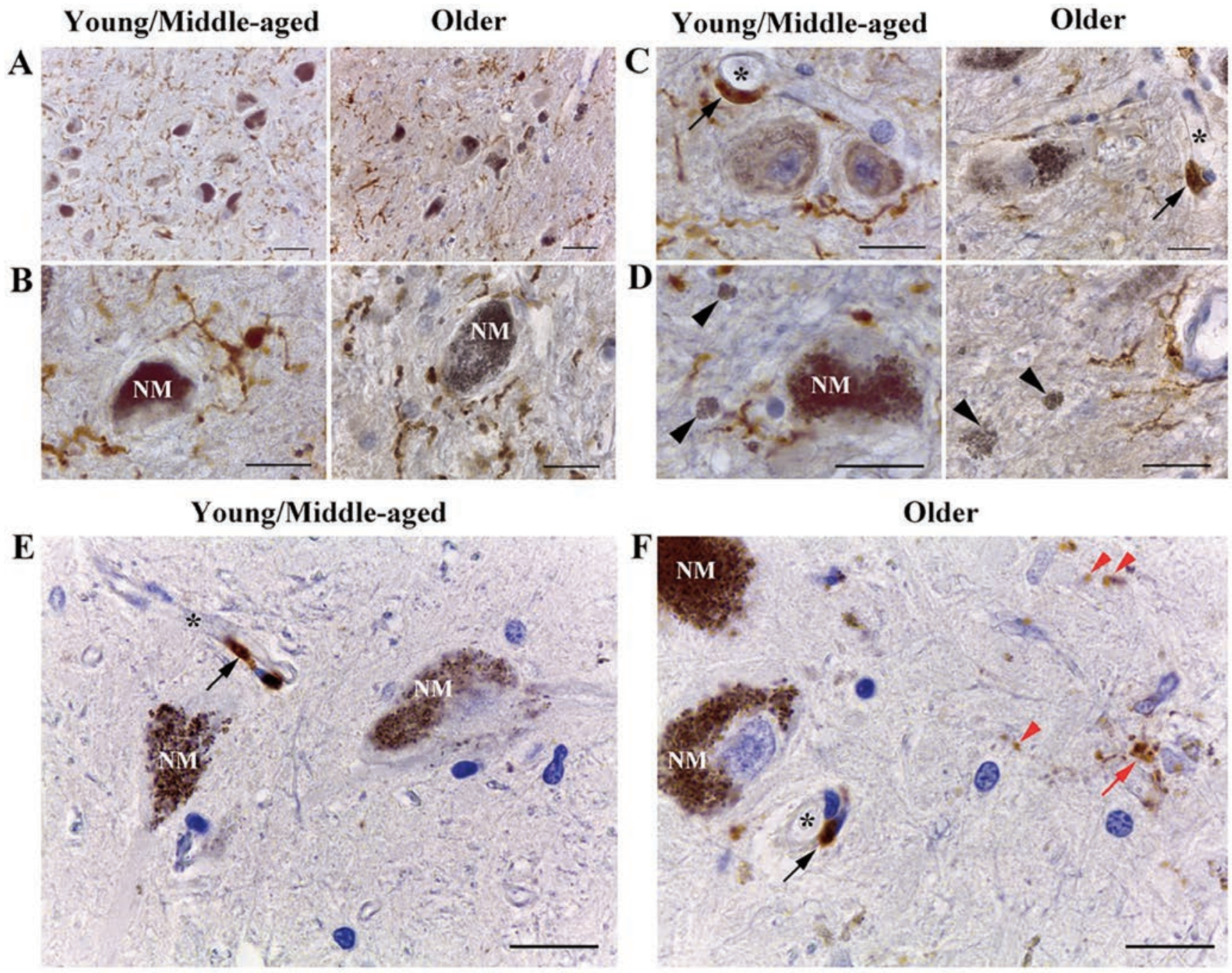

Figure 4. Microglia and macrophages in human SN pars compacta. A-D) Immunohistochemistry for Iba-1 and counterstained with hematoxylin. A) Microglia have a ramified morphology in both young/middle and older adults. No activation of microglia in SN pars compacta with age was observed. B) Iba-1-positive processes of microglia often approached the bodies of NM-containing neurons (NM - intracellular NM), contacting and surrounding them. C) In people of both age groups, the Iba-1-immunoreactive perivascular macrophages (black arrow) localized near blood vessels (asterisk - vessel lumen) were observed. D) Microglia near clusters of eNM (black arrowhead) always had ramified morphology and showed no signs of activation. E-F) Immunohistochemistry for CD68 and counterstained with hematoxylin. E) In the young/middle aged group, CD68-immunostaining were absent in the brain parenchyma. F) In the older group, discrete CD68-immunopositive granules were visualized in the parenchyma (red arrowhead); single CD68immunopositive ramified cells were detected (red arrow); in both age groups, the CD68-immunoreactive perivascular macrophages (black arrow) localized near blood vessels (asterisk - vessel lumen) were observed. Scale bar: A) $50 \mu \mathrm{m} ; \mathrm{B}-\mathrm{F}$ ) $20 \mu \mathrm{m}$. 
aged human $\mathrm{SN}$ pars compacta, suggesting that ramified microglia can perform active phagocytosis. The absence of pronounced microgliosis, as well as a pronounced loss of NM-containing neurons, may indicate the absence of neuroinflammation in human $\mathrm{SN}$ pars compacta during normal aging.

In conclusion, this study examined the structural, cytochemical and localization features of $\mathrm{NM}$ in human $\mathrm{SN}$ pars compacta. It was shown that dopaminergic neurons differ in the amount of cytoplasmic NM and the intensity of TH-immunoreactivity in an agedependent manner. Intra- and extracellular NM predominantly does not stain when using histochemical methods for detecting metals (Perl's method, Mallory's hematoxylin). The amount of extracellular NM in human SN pars compacta is positively correlated with age. No signs of neuroinflammation were found in human SN pars compacta during normal aging. We have demonstrated vast distribution of ramified microglia in $\mathrm{SN}$ of both, young/middle aged and older adults. An increase in the phagocytic activity of ramified microglia was observed with aging.

\section{References}

1. Usunoff KG, Itzev DE, Ovtscharoff WA, Marani E. Neuromelanin in the human brain: A review and atlas of pigmented cells in the substantia nigra. Arch Physiol Biochem 2002;110:257-369.

2. Simon JD, Peles D, Wakamatsu K, Ito S. Current challenges in understanding melanogenesis: bridging chemistry, biological control, morphology, and function. Pigment Cell Melanoma Res 2009;22:563-79.

3. Greggio E, Bergantino E, Carter D, Ahmad R, Costin GE, Hearing VJ, et al. Tyrosinase exacerbates dopamine toxicity but is not genetically associated with Parkinson's disease. J Neurochem 2005;93:246-56.

4. Tribl F, Arzberger T, Riederer P, Gerlach M. Tyrosinase is not detected in human catecholaminergic neurons by immunohistochemistry and Western blot analysis. In: Gerlach M, Deckert J, Double K, Koutsilieri E, editors. Neuropsychiatric disorders an integrative approach. Journal of Neural Transmission Supplementa. Vienna: Springer; 2007. p. 51-5.

5. Monzani E, Nicolis S, Dell'Acqua S, Capucciati A, Bacchella C, Zucca FA, et al. Dopamine, oxidative stress and proteinquinone modifications in Parkinson's and other neurodegenerative diseases. Angew Chem Int Ed Engl 2019;58:6512-27.

6. Vila M. Neuromelanin, aging, and neuronal vulnerability in Parkinson's disease. Mov Disord 2019;34:1440-51.

7. d'Ischia M, Prota G. Biosynthesis, structure, and function of neuromelanin and its relation to Parkinson's disease: a critical update. Pigment Cell Res 1997;10:370-6.

8. Zecca L, Costi P, Mecacci C, Ito S, Terreni M, Sonnino S. Interaction of human substantia nigra neuromelanin with lipids and peptides. J Neurochem 2000;74:1758-65.

9. Wakamatsu K, Fujikawa K, Zucca FA, Zecca L, Ito S. The structure of neuromelanin as studied by chemical degradative methods. J Neurochem 2003;86:1015-23.

10. Engelen M, Vanna R, Bellei C, Zucca FA, Wakamatsu K, Monzani E, et al. Neuromelanins of human brain have soluble and insoluble components with dolichols attached to the melanic structure. PLoS One 2012;7:e48490.

11. Double KL, Gerlach M, Schünemann V, Trautwein AX, Zecca $\mathrm{L}$, Gallorini M, et al. Iron-binding characteristics of neuromelanin of the human substantia nigra. Biochem Pharmacol 2003;66:489-94.

12. Gerlach M, Double KL, Ben-Shachar D, Zecca L, Youdim $\mathrm{MB}$, Riederer P. Neuromelanin and its interaction with iron as a potential risk factor for dopaminergic neurodegeneration underlying Parkinson's disease. Neurotox Res 2003;5:35-44.

13. Ostergren A, Annas A, Skog K, Lindquist NG, Brittebo EB. Long-term retention of neurotoxic beta-carbolines in brain neuromelanin. J Neural Transm (Vienna) 2004;111:141-57.

14. Zecca L, Zucca FA, Wilms H, Sulzer D. Neuromelanin of the substantia nigra: a neuronal black hole with protective and toxic characteristics. Trends Neurosci 2003;26:578-80.

15. Zecca L, Bellei C, Costi P, Albertini A, Monzani E, Casella L, et al. New melanic pigments in the human brain that accumulate in aging and block environmental toxic metals. Proc Natl Acad Sci USA 2008;105:17567-72.

16. Fasano M, Bergamasco B, Lopiano L. Is neuromelanin changed in Parkinson's disease? Investigations by magnetic spectroscopies. J Neural Transm (Vienna) 2006;113:769-74.

17. Schroeder RL, Gerber JP. A reappraisal of Fe(III) adsorption by melanin. J Neural Transm (Vienna) 2014;121:1483-91.

18. Karlsson O, Lindquist NG. Melanin and neuromelanin binding of drugs and chemicals: toxicological implications. Arch Toxicol 2016;90:1883-91.

19. Knorle R. Neuromelanin in Parkinson's Disease: from Fenton reaction to calcium signaling. Neurotox Res 2018;33:515-22.

20. Beach TG, Sue LI, Walker DG, Lue LF, Connor DJ, Caviness $\mathrm{JN}$, et al. Marked microglial reaction in normal aging human substantia nigra: correlation with extraneuronal neuromelanin pigment deposits. Acta Neuropathol 2007;114:419-24.

21. Ishikawa A, Takahashi H. Clinical and neuropathological aspects of autosomal recessive juvenile parkinsonism. J Neurol 1998;245:P4-9.

22. Wilms H, Rosenstiel P, Sievers J, Deuschl G, Zecca L, Lucius R. Activation of microglia by human neuromelanin is NFkappaB dependent and involves p38 mitogen-activated protein kinase: implications for Parkinson's disease. FASEB J 2003;17:500-2.

23. Zecca L, Casella L, Albertini A, Bellei C, Zucca FA, Engelen M, et al. Neuromelanin can protect against iron-mediated oxidative damage in system modeling iron overload of brain aging and Parkinson's disease. J Neurochem 2008;106:1866-75.

24. Zhang W, Phillips K, Wielgus AR, Liu J, Albertini A, Zucca FA, et al. Neuromelanin activates microglia and induces degeneration of dopaminergic neurons: implications for progression of Parkinson's disease. Neurotox Res 2011;19:63-72.

25. Zhang W, Zecca L, Wilson B, Ren HW, Wang YJ, Wang XM, et al. Human neuromelanin: an endogenous microglial activator for dopaminergic neuron death. Front Biosci (Elite Ed) 2013;5:1-11.

26. Carballo-Carbajal I, Laguna A, Romero-Giménez J, Cuadros T, Bové J, Martinez-Vicente M, et al. Brain tyrosinase overexpression implicates age-dependent neuromelanin production in Parkinson's disease pathogenesis. Nat Commun 2019;10:973.

27. Depboylu C, Schäfer MK, Arias-Carrion O, Oertel WH, Weihe E, Höglinger GU. Possible involvement of complement factor $\mathrm{C} 1 \mathrm{q}$ in the clearance of extracellular neuromelanin from the substantia nigra in Parkinson disease. J Neuropathol Exp Neurol 2011;70:125-32.

28. Lawana V, Um SY, Foguth RM, Cannon JR. Neuromelanin formation exacerbates HAA-induced mitochondrial toxicity and mitophagy impairments. Neurotoxicology 2020;81:147-60.

29. Prota G, d'Ischia M. Neuromelanin: a key to Parkinson's disease. Pigment Cell Res 1993;6:333-5.

30. Xu S, Chan P. Interaction between neuromelanin and alpha-synuclein in Parkinson's disease. Biomolecules 2015;5:1122-42.

31. Korzhevskii DE, Sukhorukova EG, Kirik OV, Grigorev IP. Immunohistochemical demonstration of specific antigens in the human brain fixed in zinc-ethanol-formaldehyde. Eur J 
Histochem 2015;59:2530.

32. Mallory FB. Pathological technique: a practical manual for workers in pathological histology including directions for the performance of autopsies and for microphotography. Philadelphia and London: W.B. Saunders Co.; 1938.

33. Culling CFA, Allison RT, Barr WT. Cellular pathology technique. Oxford: Butterworth-Heinemann; 1985.

34. Korzhevskii DE, Grigor'ev IP, Sukhorukova EG, Guselnikova VV. Immunohistochemical characteristics of neurons in the substantia nigra of the human brain. Neurosci Behav Physi 2019;49:109-14.

35. Sulzer D, Mosharov E, Talloczy Z, Zucca FA, Simon JD, Zecca L. Neuronal pigmented autophagic vacuoles: lipofuscin, neuromelanin, and ceroid as macroautophagic responses during aging and disease. J Neurochem 2008;106:24-36.

36. Sulzer D, Bogulavsky J, Larsen KE, Behr G, Karatekin E, Kleinman $\mathrm{MH}$, et al. Neuromelanin biosynthesis is driven by excess cytosolic catecholamines not accumulated by synaptic vesicles. Proc Natl Acad Sci USA 2000;97:11869-74.

37. Zecca L, Pietra R, Goj C, Mecacci C, Radice D, Sabbioni E. Iron and other metals in neuromelanin, substantia nigra, and putamen of human brain. J Neurochem 1994;62:1097-101.

38. Moreno-García A, Kun A, Calero M, Calero O. the neuromelanin paradox and its dual role in oxidative stress and neurodegeneration. Antioxidants (Basel) 2021;10:124.

39. Double KL, Dedov VN, Fedorow H, Kettle E, Halliday GM, Garner B, et al. The comparative biology of neuromelanin and lipofuscin in the human brain. Cell Mol Life Sci 2008;65:1669-82.

40. Riga D, Riga S, Halalau F, Schneider F. Brain lipopigment accumulation in normal and pathological aging. Ann NY Acad Sci 2006;1067:158-63.

41. Jellinger K, Paulus W, Grundke-Iqbal I, Riederer P, Youdim MB. Brain iron and ferritin in Parkinson's and Alzheimer's diseases. J Neural Transm Park Dis Dement Sect 1990;2:327-40.

42. Zecca L, Gallorini M, Schünemann V, Trautwein AX, Gerlach $\mathrm{M}$, Riederer $\mathrm{P}$, et al. Iron, neuromelanin and ferritin content in the Substantia Nigra of normal subjects at different ages: consequences for iron storage and neurodegenerative processes. J Neurochem 2001;76:1766-73.

43. Jellinger K, Kienzl E, Rumpelmair G, Riederer P, Stachelberger H, Ben-Shachar D, et al. Iron-melanin complex in substantia nigra of parkinsonian brains: an x-ray micro- analysis. J Neurochem 1992;59:1168-71.

44. Kienzl E, Jellinger K, Stachelberger H, Linert W. Iron as catalyst for oxidative stress in the pathogenesis of Parkinson's disease? Life Sci 1999;65:1973-6.

45. Sukhorukova EG, Grigoriev IP, Kirik OV, Alekseeva OS, Korzhevskii DE. Intranuclear localization of iron in neurons of mammalian brain. J Evol Biochem Physiol 2013;49:370-2.

46. Korzhevskii D, Sukhorukova EG, Kirik OV, Grigorev IP. A Cytochemical study of iron-containing intranuclear structures of the human substantia nigra dopaminergic neurons with special emphasis on the Marinesco bodies. Opera Med Physiol 2017;3:99-107.

47. Reinert A, Morawski M, Seeger J, Arendt T, Reinert T. Iron concentrations in neurons and glial cells with estimates on ferritin concentrations. BMC Neurosci 2019;20:25.

48. Roschzttardtz H, Grillet L, Isaure M-P, Conéjéro G, Ortega R, Curie C, et al. Plant cell nucleolus as a hot spot for iron. J Biol Chem 2011;286:27863-6.

49. Surguladze N, Thompson KM, Beard JL, Connor JR, Fried MG. Interactions and reactions of ferritin with DNA. J Biol Chem 2004;279:14694-702.

50. Honda K, Smith MA, Zhu X, Baus D, Merrick WC, Tartakoff AM, et al. Ribosomal RNA in Alzheimer disease is oxidized by bound redox-active iron. J Biol Chem 2005;280:20978-86.

51. Zecca L, Fariello R, Riederer P, Sulzer D, Gatti A, Tampellini D. The absolute concentration of nigral neuromelanin, assayed by a new sensitive method, increases throughout the life and is dramatically decreased in Parkinson's disease. FEBS Lett 2002;510:216-20.

52. Jyothi HJ, Vidyadhara DJ, Mahadevan A, Philip M, Parmar SK, Manohari SG, et al. Aging causes morphological alterations in astrocytes and microglia in human substantia nigra pars compacta. Neurobiol Aging 2015;36:3321-33.

53. Kanaan NM, Kordower JH, Collier TJ. Age-related changes in glial cells of dopamine midbrain subregions in rhesus monkeys. Neurobiol Aging 2010;31:937-52.

54. Jurga AM, Paleczna M, Kuter KZ. Overview of general and discriminating markers of differential microglia phenotypes. Front Cell Neurosci 2020;14:198.

55. Hopperton KE, Mohammad D, Trépanier MO, Giuliano V, Bazinet RP. Markers of microglia in post-mortem brain samples from patients with Alzheimer's disease: a systematic review. Mol Psychiatry 2018; 23:177-98.

Received for publication: 31 May 2021. Accepted for publication: 22 July 2021.

This work is licensed under a Creative Commons Attribution-NonCommercial 4.0 International License (CC BY-NC 4.0).

(C) Copyright: the Author(s), 2021

Licensee PAGEPress, Italy

European Journal of Histochemistry 2021; 65(s1):3283

doi:10.4081/ejh.2021.3283 